\title{
The Tissue Reactions and Changes of a Surface of Various Metal Implants after Their Introduction in a Bone Tissue in Experiment
}

\author{
Michael S. Toder ${ }^{1}$, Andrew I. Shevela ${ }^{2}$, Alexander A. Shevela ${ }^{1,3}$, Paul A. Zheleznyi ${ }^{3}$, \\ Anna P. Zheleznaia ${ }^{3}$, Igor V. Mayborodin' ${ }^{2}$ \\ ${ }^{1}$ International Center of Implantology of iDent, Novosibirsk, Russia \\ ${ }^{2}$ Center of New Medical Technologies, Institute of Chemical Biology and Fundamental Medicine, Russian \\ Academy of Science, Siberian Branch, Novosibirsk, Russia \\ ${ }^{3}$ Novosibirsk State Medical University, Novosibirsk, Russia \\ Email: imai@mail.ru
}

Received 27 January 2016; accepted 22 February 2016; published 25 February 2016

Copyright (C) 2016 by authors and Scientific Research Publishing Inc.

This work is licensed under the Creative Commons Attribution International License (CC BY). http://creativecommons.org/licenses/by/4.0/

(c) () Open Access

\section{Abstract}

Screw metal implants (3S, Israel) with rough or smooth polished surface were introduced in a tibial proximal condyle of not purebred rabbits. The condition of surrounding tissues in 2 and 6 months after implantation was compared by light microscopy and X-ray methods. Within 6 months after operation the considerable distinctions of radiological and morphological data were revealed not. 2 months later after introduction of implants with a rough surface the effort enclosed for its twisting is, much more, than for removal of the polished product. However, stability of fixing of implants was practically made even at 6 months. On remote rough implants there is a set of tissue scraps whereas on products with a smooth surface the tissue remains were much less. Surrounding tissues strongly join a rough surface, grow into cavities, and during removal of such products there is a considerable trauma of tissues round an implantation place. Smooth implants have the smaller area of contact with organism tissues, they are fixed due to bicortical implantation, during removal easily get out and don't break off surrounding tissues. The signs of inflammation and formation of merged multinuclear macrophages were not found at all cases, which give evidence to the inertness of material of the mentioned articles for living organism. In some observations however and by implantation of the rough article and by introduction of polished implants, metal particles were found, but after use of the foreign body with grit-blasted treatment of surface metal was found more frequently, and its fragments had larger volume. 


\section{Keywords}

\section{Implantation of Metals Intra Bone, Durability of Implant Fixing, Isolation of Foreign Bodies, Metal Fragments in Tissues, Features of Implant Removal}

\section{Introduction}

The study of organism response to various artificial materials is of considerable importance in relation to the development of implants in traumatology and orthopedics, in rehabilitation medicine and dentistry. The researches of last years showed that tissues of biological systems including the tissues of human body possess specific features: they are indestructible at significant deformations under multiple loads and vibrations and restore the initial form after cancellation of load, showing high elasticity. The compatibility of living and non-living tissues presupposes the absence of overloads and macro-shifts on the implant-tissue partition surface. Due to it many implants are made of metals, which wide application is conditional on their firmness, durability, corrosion and wear-resistance [1].

The requirement of biochemical compatibility and implants fixation and body tissues can be resolved satisfactory, if one applies the materials with rough surface, which is able to fix firmly to the living tissue. Two linking methods between implant and living tissue are created there: a mechanical adhesion due to the formation (growing in) of the tissue into the implant pores and chemical adhesion due to the interrelation of the tissue with implant content elements. The sort of material and nature of surface has an influence on reactions going on at living tissue-implant partition line [1] [2].

Tissue response to the intrusion of a foreign body usually includes inflammation, vascular responses and its separation by connective tissue [3]-[8]. Minimal expression of inflammatory process (low number of leukocytes in the tissue, weak expression of vascular response), absence of giant cells of foreign bodies in metal implants enclosing tissues, insufficient thickness of connective tissue capsule for all terms of research indicates the inertness of such materials for living organism, high compatibility of many metals with biological tissues [1] [2].

The objective of the study was defined based on the mentioned above: To compare tissue changes after implantation into the bone of metal implants with rough or smooth surface.

\section{Materials and Methods}

The tests of new articles, their comparison with the applicable articles, testing the new methods of implantation is carried out on experimental animals. The best suitable for this goal are rabbits, specifically proximal condyle of their shin bone, the structure of which corresponds to some human bones very closely. Besides, this part of the extremity of these animals are easy accessible. Due to it, very many researchers use this model for experimental implantation.

Metal articles with various type of surface: rough dental implant with grit blasting and acid processing (3S, Israel) and smooth polished article for bicortical dental implantation (3S, Israel) were implanted into proximal condyle of shin bone of 10 non-pedigreed rabbits.

All manipulations with the animals were not connected with distress and carried out in compliance with "Regulations on the works using experimental animals". The implantation was carried out with all aseptic and antiseptic rules in terms of sterile operating room under general anesthesia based on total intravenous anesthesia by propofol. At the initial stage marginal vein of auricle was punctured and catheterized by $24 \mathrm{G}$ catheter, which were fixed by adhesive plaster. The intravenous premedication was carried out: atropine sulphate $0.1 \%-0.22$ $0.27 \mathrm{mg} / \mathrm{kg}$; benadryl $1 \%-4.6-5.2 \mathrm{mg} / \mathrm{kg}$; droperidol $0.25 \%-1.25 \mathrm{mg}$; ketorolac trimetamine $1 \%-10 \mathrm{mg}$. Propofol $1 \%-15 \mathrm{mg} / \mathrm{kg}$ was used intravenously as anesthesia induction, propofol $1 \%-25-30 \mathrm{mg} / \mathrm{kg} / \mathrm{hour}$ intravenopusly was also used for support of anesthesia. The infusion therapy was effected by $0.9 \%$ sodium chloride solution of $15-25 \mathrm{ml} / \mathrm{kg}$, depending on the expression of blood loss. The respiratory support when necessary was carried out by mask method with $100 \%$ oxygen insuflation.

Removal of hair was carried out by surgical scissors on the place of supposed surgical invasion on both extremities at knee joints. After manipulations the skin was processed by alcohol, the operation field was covered with a sterile nap with a hole. 
For implantation of articles with rough surface the left extremity was chosen, for implantation of smooth implants - the right extremity was chosen. It is necessary to note that the introduction of each implant into the same extremity of different animals firstly gives an opportunity to accelerate the implantation procedure itself, because there is no need to register what animal and what extremity an article was implanted into; secondly, it makes further observation and examination of animals easier.

The sequence of manipulations for implantation:

1) The skin section was made by disposable scalpel along the front line a little bit below the knee joint.

2) The tissues were moved apart to the periosteum by the blunt method (closed branches of the forceps).

3) The surface of proximal condyle of shin bone was exposed by the rasp at the front-medial side.

4) The hole in chosen part was made by the dentist's drill up to the “downfall”. 2 bores of increasing diameter are used.

5) The implant was screwed into the made hole by hand and then by the spanner with dynamometric scale, allowing to control the force created. The implant head goes beyond the bone surface for not more than $1 \mathrm{~mm}$.

6) Operative wound was taken in by vicryl 5-0 on each layer.

7) Skin stitches were treated by $5 \%$ alcohol-iodine solution.

The animals get rid of stitches by themselves within 1 - 2 weeks after the operation, there were no visual signs of acute inflammatory process. In most cases for all times the implants were placed on introduction place: in proximal condyle of tibial bone.

All rats were X-rayed every week to control the location of foreign bodies and early determination of potential complications. There are one case of osteomyelitis development after the implantation in the group as a whole and one case of rough and polished implants (different animals). The break of the extremity at the implantation place was detected during the last examination, the complication was found 1 week after the operation and it probably occurred at the time of or immediately after the implantation. Such animals were rejected and did not participate in further studies.

For morphologic research of rabbits, the animals were taken out of the experiment 2 or 6 months after the operation by overdose of inhalation ether narcosis. Due to the fact that it was necessary to evaluate the conditions of surrounding tissues and to determine suppurative complications around before removal of foreign bodies, it is reasonable to preliminary remove the entire skin from the extremity from inguinal fold to down to the ankle. In cases, when the implant head was covered by tissues, it was released by the scalpel. Then the spanner with dynamometric scale was put onto it and the implant was screwed out step by step, registering the force at the beginning of its turn. After removal of foreign body a hole with even edges remained in tissues with traces of thread pane.

All manipulations with the object leading to its damage should be better carried out after its fixation to exclude doubts in life-time changes, and further decalcification makes the bone weak and facilitates the excision of the fragment required for further processing, besides, the dehydrogenated bone does not crack. For further morphological research the shin bone after maximal removal of soft tissues was fixed in 4\% para-formaldehyde solution on phosphate buffer not less than 24 hours. Then the bone was decalcified by the solution "Biodec R" (Bio Optica Milano, Italy) within 7 days, which damages the tissues less compared with acid decalcification. The duration of processing was determined trying to puncture the bone by dissecting needle far from the hole after removal of the implant. Decalcification was stopped after the bone became soft and elastic and was easy to cut by scalpel. Further, the bone fragments with the hole were dehydrated constantly mixing up in ethanol with increasing concentration, were clarified in xylene and enclosed into plasticized paraffin. The sections of 5 - 7 $\mu \mathrm{m}$ thick were stained with hematoxylin and eosin and examined by the light microscope Axioimager M1 (Zeiss, Germany) at magnification to 1200 times.

Statistical data processing was carried out by means of application statistic program MS Excel 7.0 (Microsoft, USA), arithmetic mean and error of arithmetic mean (standard deviation) were determined. The reliability of differences of compared mean values was determined on the basis of Student's test. As reliable was considered the difference between compared rows with confidence level of 95\% and more. The calculations considered that the distribution of researched features was near to normal.

\section{Results}

From radiological point of view the implants were placed at introduction places for all the times: in proximal 
condyle of shin bone. It is necessary to note that at the places where implant heads were (contact with periosteum) in 4 weeks after the operation some radiological signs of its irritation (thickening) appear. After 2 weeks these signs became more expressed (Figure 1(a), Figure 1(b)). After 6 months the response of periosteum to the foreign body did not change, after removal of implants radiological parts of decreased density remain after the removal of implants. The increase of density is noted in external areas of condyle, where the implant was introduced —its head; and at the opposite side — where the implant end rests.

2 months after the implantation a double difference was found between the compared articles during processing of statistic data on the force applied to the unscrewing implants: $46 \pm 8.94 \mathrm{Ncm}$ has the rough implant, and $23 \pm 18.2 \mathrm{Ncm}$ has the polished implant. 6 months after the operation the force of the unscrewing implants was equal to $67.5 \pm 9.57 \mathrm{Ncm}$ (rough implant) and $52 \pm 5.42 \mathrm{Ncm}$ (smooth implant) respectively. All differences were however unreliable due to a big value of error of arithmetic means.

Multiple fragments of tissues were found macro- and microscopically at the removed implants with gritblasted surface and further itching (Figure 2(a)). The edge of proximal condyle of shin bone, immediately adjacent to the implant had no notable changes during microscopic research. It can be noted only structures of callus in some parts (Figure 2(b)). The formation of a thin strip of compact bone tissue was noted in bone marrow at places of its contact with the implant material. But in some cases, the foreign body was separated from bone marrow structures by fibrous connective tissue, closer to metal it was separated by dense and further by friable tissue. The bone marrow itself both red and yellow had no visible pathologic changes. Metal fragments of different size without surrounding inflammatory reaction were found in bone tissue itself, immediately adjacent to the foreign body, and in bone marrow (Figure 2(c)).

There were much less fragments of tissues on articles with smooth surface (Figure 3(a)). In some cases clear traces from the screw thread edge of the implant in condyle tissue immediately after introduction of the foreign body by little zoom of the light microscope, the bone sides had cicatricle changes, and the cavities formed after removal of the article was filled in with blood or fibrin. It is necessary to note that thinning of the whole bone in limited parts was found, which can be referred to the installation of the screw-thread at this place (edges of external diameter of the bolt) or with long-term pressure of the foreign body on the bone tissue (Figure 3(b)).

No pathologic changes were found both in red and yellow bone marrow inside the bone at the place of introduction of the foreign body. Along the implant the bone marrow was separated by fibrous connective tissue: closer to metal - by a dense, further — by friable or thin strip of compact bone. Sometimes hemorrhages were present in such connective tissue. In some cases it was practically impossible to differ a dense friable connective tissue from a rough friable tissue of the bone. Probably, these types of connective tissue separating the foreign body and bone marrow structures turn into each other (Figure 3(c)).

It is also necessary to note the presence of metal particles in connective tissue, separating the bone marrow from the surface of smooth implant, and metal was present close to friable connective tissue and close to rough fibrous tissue, similar to the bone. But the number of such metal fragments and their size was much less than it was after introduction of article with rough surface (Figure 3(c)).
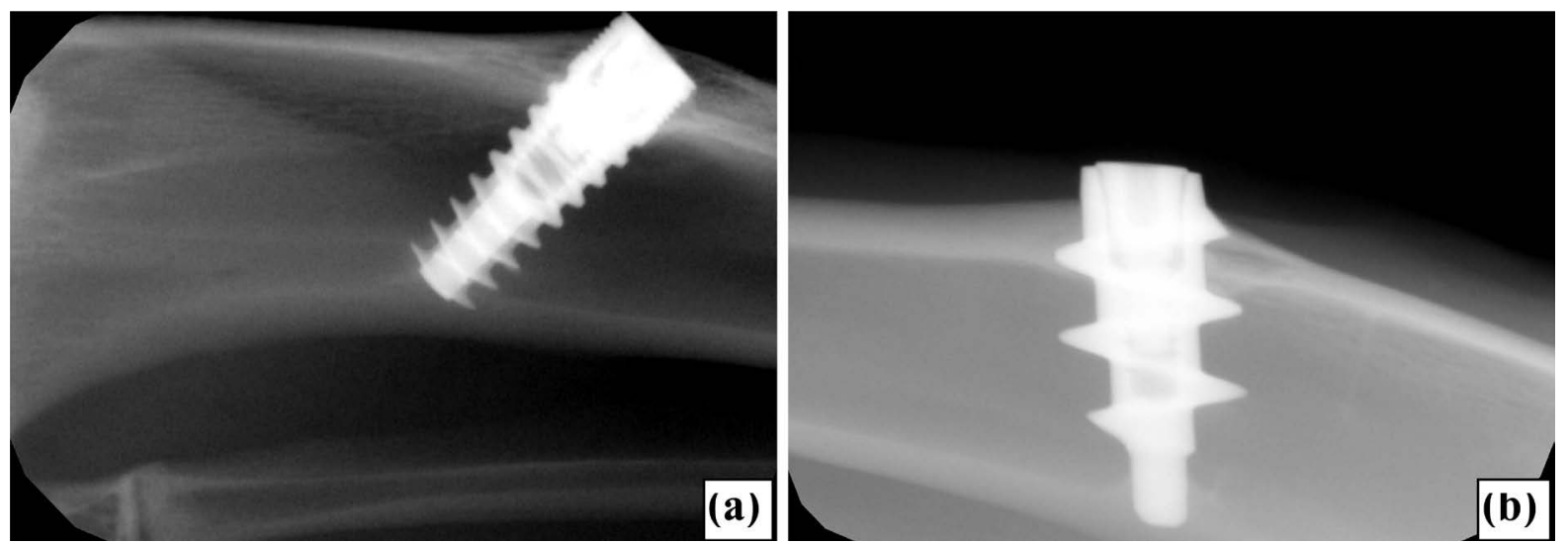

Figure 1. By results of radiological research in 2 months after introduction the implants with a rough (a) and smooth (b) surface is located in a condyle of a tibial bone, there is a thickening of a periosteum and increase in density of a bone in the field of a product head. 

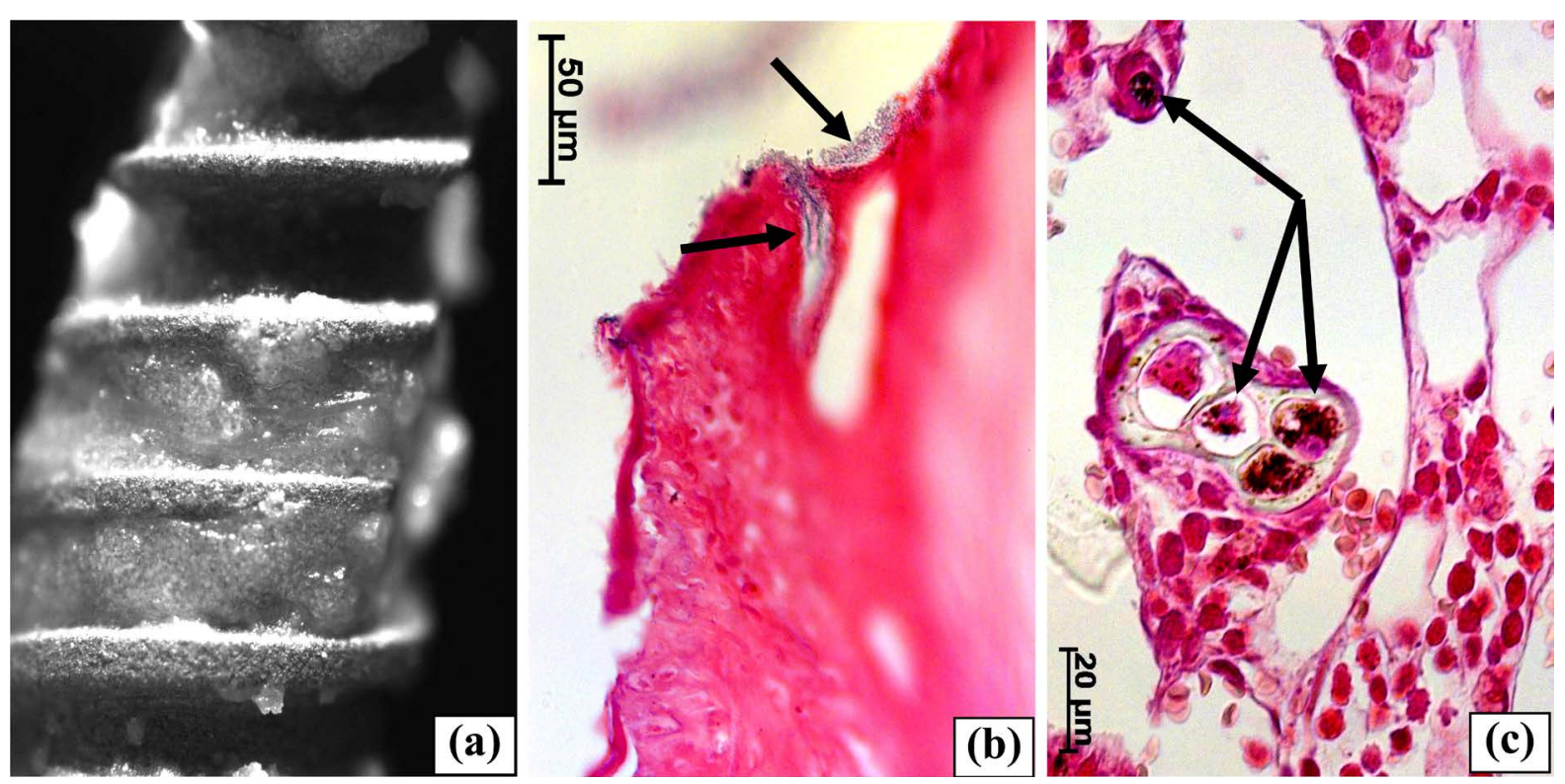

Figure 2. A result of implantation of products with a rough surface. (a) Tissue fragments on various structures of remote implant in 6 months after surgical intervention; (b) Structures of a bone callosity in proximal condyle tissue of a tibial bone in 2 months after introduction of an implant, in certain cases at a bone there are particles of metal (arrow), by hematoxylin and eosin staining; (c) The encapsulated particles of metal (arrow) in red bone marrow in 6 months after introduction of an product in a proximal condyle of a tibia, by hematoxylin and eosin staining.
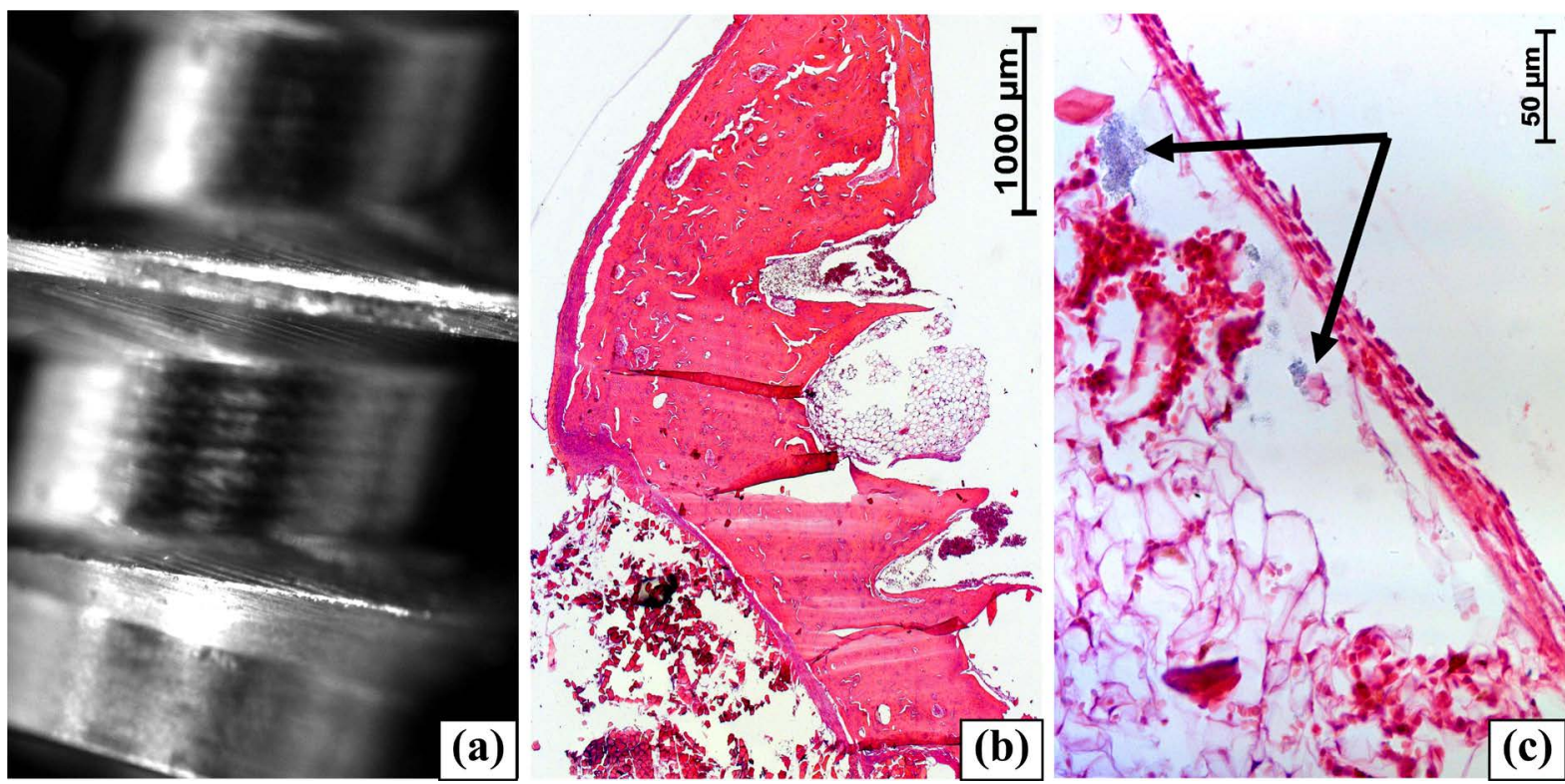

Figure 3. A result of introduction of polished implants. (a) Fragments of tissues on the implant surface in 6 months after operation are practically absent; (b) 2 months later after implantation in a place of product side contact with a bone of condyle the traces of a screw thread are located, the bone tissue has cicatricial changes; the bone cavities created after removal of an implant are filled with fibrin or blood; by hematoxylin and eosin staining; (c) Connective tissue with haemorrhage and metal particles (arrow) in marrow in the region of the polished foreign matter in 2 months after surgical intervention, by hematoxylin and eosin staining.

\section{Discussion}

The surrounding tissues are firmly connected to the rough surface of the implants, they grow into pores, and when such articles are unscrewed, the significant traumatization of tissues surrounding the place of implantation. 
Smooth polished implants have less contact area with the tissues of organism, they are fixed due to bicortical implantation, they are easy unscrewed by removal and do not break the surrounding tissues. Because of that there were many fragments of tissues on removed implants with gritblasted surface, and there were almost no fragments of tissues on articles with smooth surface.

2 months after the implantation a double difference of unscrewing force was found between two compared articles, implant with rough surface was fixed in tissues more firmly. After 6 months, however, durability of implants fixation increased in both cases, but this index for the article with smooth surface increased more significantly. To this time the mean values in the group as a whole became almost equal. Probably, the implant with rough surface is fixed in tissues quicker due to its growing into the pores of articles itself. By introduction of the polished material, this process goes a bit slower, but it takes some time however. For rabbits it takes 6 months and leads as a result to the firm fixation of implanted metal.

The decrease of bone thickness on limited parts at the place of contact with edges of implant thread is probably conditional on atrophy processes firstly and on reorganization of bone tissue, secondly. There are many reports in literature about atrophic and even necrotic changes in tissues contacting with foreign bodies [2] [9]-[11].

Atrophic changes in contact places of implants with bone can be explained from various points:

1) Expressed inflammatory reaction. An acute and then chronic inflammatory process is going on in tissues around the foreign body, incurred firstly by surgical intervention, then-by the foreign body itself. The surrounding tissues are involved into this inflammatory reaction and become atrophic and sclerotic step by step.

2) The pressure of the foreign body onto the tissues immediately contacting with it. Long-term pressure of implants leads to the trauma of surrounding tissues which is followed by inflammatory reaction. Feeding blood vessels are probably squeezed with the bone and bone marrow structures.

3) The trauma incurred by the foreign body itself. The direct damage of surrounding tissues by implants is not excluded, e.g. by their sharp edges [3] [4]. It leads to chronic inflammation (long-term trauma) and gradual atrophy.

4) The atrophy of tissues is possible due to their denervation that occurs by preparation of implantation place, when the surface of proximal condyle of shin bone was uncovered by the rasp.

The impact of all mentioned reasons on the state of bone tissue in place of implant introduction. Continuous irritation of the bone by a foreign body also facilitates the formation of callus on some parts and appearance of radiological signs of periosteum response.

Any foreign body is separated from the organism by connective tissue capsule and this is a normal response of the tissue [3]-[5]. The most probably is that because of it the founded separation of implants from bone marrow structures occurred by various types of fibrous connective tissue: closer to metal-by dense, further-by friable. Sometimes a thin strip of compact bone was formed there, that is also one of the types of connective tissue. Probably, these types of connective tissue separating the foreign body and bone marrow structures turn into each other in some parts.

At the place of contact of living tissues with the implant, the tissues respond to the foreign body. Probably, the implants immediately after their introduction into the bone firstly initiate acute inflammatory reaction due to surgical trauma and direct interaction of living tissues with the firm non-elastic non-living substance. Acute inflammatory process is gradually changed by chronic one and as it goes out slowly the full union of implant surface with the bone occurs.

By removal of the article, such dense connections of bone tissue with metal break and the cavities in the bone (traces from the edges of screw-thread of the implant) are filled in with blood or fibrin. Because of the same, hemorrhages occurred in connective tissue, separating the foreign body from the bone narrow.

Besides, because even firm foreign bodies destruct by protection systems of human body, the processes of implant degradation go on along the borderline of the implant. Large and small fragments of any foreign bodies are "cut off" by phagocytes [3]-[5] [12], enveloped by connective tissue, absorbed by macrophages (giant cells of foreign bodies), containing among other lysosomal fragments metalproteinase, and are transported into other organs for elimination. Because of that small particles of implants appear in the tissues around the implants. It is natural that particles of rough article surface are easy fragmented and separated from the foreign body [5].

That means the more inert for the organism is the material of implant, the less intensive response of microphage system will be (and vice-versa). Consequently, absence of inflammatory reaction, giant cells of foreign body, connective tissue capsule in surrounding tissues for all times of research give evidence for inertness of the material of articles with rough and with smooth surface for living organism, for compatibility of their materials 
with tissues. Probably, the material influences on the nature of relationship of the foreign body with living tissues in the first instance, and then the type of surface.

\section{Conclusion}

Therefore, within 6 months after operation the considerable distinctions of radiological data were revealed not. 2 months later after introduction of implants with a rough surface the effort enclosed for its twisting is, much more, than for removal of the polished product. However, stability of fixing of implants was practically made even at 6 months. On remote rough implants there is a set of tissue scraps whereas on products with a smooth surface the tissue remains were much less. Surrounding tissues strongly join a rough surface, grow into cavities, and during removal of such products there is a considerable trauma of tissues round an implantation place. Smooth implants have the smaller area of contact with organism tissues, they are fixed due to bicortical implantation, during removal easily get out and don't break off surrounding tissues. The signs of inflammation and formation of merged multinuclear macrophages were not found at all cases, that gives evidence to the inertness of material of the mentioned articles for living organism. In some observations however and by implantation of the rough article and by introduction of polished implants, metal particles were found, but after use of the foreign body with grit-blasted treatment of surface metal was found more frequently, and its fragments had larger volume. There are also no expressed differences between the state of surrounded tissues in 2 and 6 months after introduction of each article.

\section{References}

[1] Günter, W.E., Dambayev, G.T., Sysolyatin, P.G., et al. (1998) Medical Materials and Implants with Shape Memory. Publishing House Tomsk Un-That, Tomsk, 487 p.

[2] Maĭborodin, I.V., Iakushenko, V.K. and Maĭborodina, V.I. (2002) Interaction of Nickelide-Titanium Implant with Tissues in Human. Arkhiv Patologii, 64, 50-52.

[3] Maĭborodin, I.V., Shevela, A.I., Matveeva, V.A., et al. (2012) Morphological Tissue Changes after the Implantation of Elastic Lamellar Foreign Bodies in the Experiment. Morfologiia, 141, 54-60.

[4] Maiborodin, I.V., Kuznetsova, I.V., Beregovoy, E.A., et al. (2013) Tissue Reactions during the Degradation of Polylactide Implants in the Body. Morfologiia, 143, 59-65.

[5] Maĭborodin, I.V., Shevela, A.I., Kuznetsova, I.V., et al. (2013) Tissue Responses to Silicone Materials in the Body. Arkhiv Patologii, 75, 28-33.

[6] Miro-Mur, F., Hindié, M., Kandhaya-Pillai, R., et al. (2009) Medical-Grade Silicone Induces Release of Proinflammatory Cytokines in Peripheral Blood Mononuclear Cells without Activating T Cells. Journal of Biomedical Materials Research Part B: Applied Biomaterials, 90, 510-520. http://dx.doi.org/10.1002/jbm.b.31312

[7] Rodriguez, A. and Anderson, J.M. (2010) Evaluation of Clinical Biomaterial Surface Effects on T Lymphocyte Activation. Journal of Biomedical Materials Research Part A, 92, 214-220. http://dx.doi.org/10.1002/jbm.a.32362

[8] Rodriguez, A., Meyerson, H. and Anderson, J.M. (2009) Quantitative in Vivo Cytokine Analysis at Synthetic Biomaterial Implant Sites. Journal of Biomedical Materials Research Part A, 89, 152-159.

[9] Gabriel, S.E., Woods, J.E., O’Fallon, W.M., et al. (1997) Complications Leading to Surgery after Breast Implantation. New England Journal of Medicine, 336, 677-682. http://dx.doi.org/10.1056/NEJM199703063361001

[10] Kanhai, R.C., Hage, J.J., Karim, R.B. and Mulder, J.W. (1999) Exceptional Presenting Conditions and Outcome of Augmentation Mammaplasty in Male-to-Female Transsexuals. Annals of Plastic Surgery, 43, 476-483. http://dx.doi.org/10.1097/00000637-199911000-00003

[11] Tebbetts, J.B. (2002) A System for Breast Implant Selection Based on Patient Tissue Characteristics and Implant-Soft Tissue Dynamics. Plastic and Reconstructive Surgery, 109, 1396-1409. http://dx.doi.org/10.1097/00006534-200204010-00030

[12] Greene, W.B., Raso, D.S., Walsh, L.G., et al. (1995) Electron Probe Microanalysis of Silicon and the Role of the Macrophage in Proximal (Capsule) and Distant Sites in Augmentation Mammaplasty Patients. Plastic and Reconstructive Surgery, 95, 513-519. http://dx.doi.org/10.1097/00006534-199503000-00012 